\title{
Deviations of the Surface Brightness Distribution in Isolated Elliptical Galaxies
}

\author{
Omar Basil Mohammad Saleh \\ Department of Physics / College of Education \\ University of Mosul
}

Received

$11 / 03 / 2012$
Accepted

$02 / 05 / 2012$

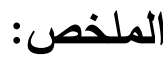

تم في هذا البحث، دراسة منحنيات السطوع السطحي لـ 48 مجرة اهليجية معزولة. حيث

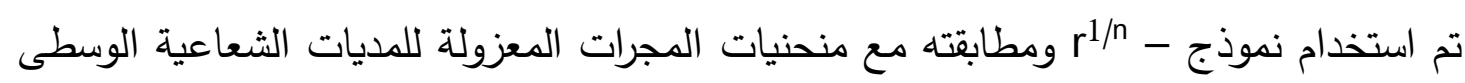
0.1 $0 .\left(\frac{R}{R_{e}}\right) \leq 1.5$ منحنيات الانحرافات بين الأرصاد والنموذج المعتمد. وبينت النتائج إن الانحرافات هي مماثلة

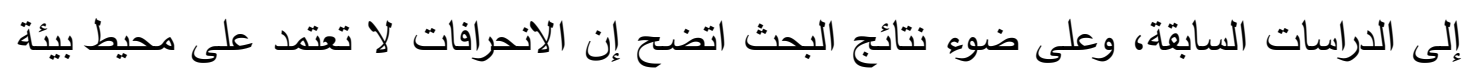

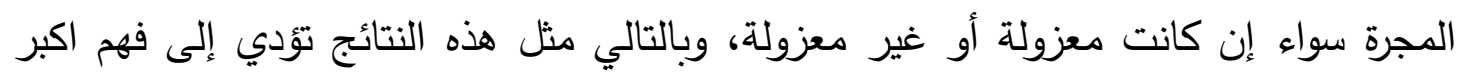
لتطور وتركيب البنية للمجرات الاهليجية.

\begin{abstract}
:
In this paper, we present the surface brightness profiles of 48 isolated elliptical galaxies. we have adopted the $\mathrm{r}^{1 / \mathrm{n}}-$ law to the observed surface brightness profiles of the adopted sample of isolated elliptical galaxies for the intermediate radial range $0.1 \leq\left(\frac{R}{R_{e}}\right) \leq 1.5$. From best fitting profile. The results showed that the deviations are similar to previous studies, and the deviations do not depend on the surrounding environment of the galaxies, whether that were an isolated or not. Such results might be very useful to understand the structure and evolution of elliptical galaxies.
\end{abstract}

\section{Introduction:}

A more recent result is that the surface brightness profile of elliptical galaxies frequently deviates from the $r^{1 / 4}-$ law, the "universal" 
$\mathrm{r}^{1 / \mathrm{n}}$ - law (equation 1) has provided the best fit in many instances (Caon et al. 1993). The parameter $n$ takes values typically between 1 and 15 , and is related to the effective radius and to the absolute magnitude of the galaxy (Trujillo et al. 2001).

$$
\mu(R)=\mu_{e}+1.0857 b_{n}\left[\left(\frac{R}{R_{e}}\right)^{1 / n}-1\right]
$$

where $\mu_{e}$ is the surface brightness at the effective radius $R_{\mathrm{e}}$ that encloses half of the total light of the model, the constant $b_{n}$ is defined in terms of the parameter $\mathrm{n}$ which describes the 'shape' of the light profile, a good approximation is $b_{n} \approx 2 n-0.327$ for $1 \leq n \leq 15$ (Trujillo et al., 2001).

The study of Isolated elliptical galaxies important to driven the processes of the structure and evolution of galaxies (Pamela et al., 2004). Isolated elliptical galaxies provide a useful control sample, typically found in highly clustered regions of the universe. There are two kinds of isolated elliptical, low environmental density and close companions (Jack, 2009). Karachentseva (1973) was chosen his catalogue (KIG) about 1000 isolated galaxies with $m_{B}<15.7$ from the Zwicky et al. (1957) catalogue which is known to be biased against surface brightness galaxies. Colbert et al. (2001) has studied 30 isolated early-type galaxies, selected from the Third Reference Catalog (RC3; de Vaucouleurs et al. 1991) catalogue. Galaxies were selected to have no catalogued neighbours within $1 \mathrm{~h}^{-1} 100 \mathrm{Mpc}$ (where $h=H_{0} / 100 \mathrm{~km} \mathrm{~s}^{-1} \mathrm{Mpc}^{-1}$ ) and $\pm 1000 \mathrm{~km} \cdot \mathrm{s}^{-1}$. However, some of the target galaxies themselves had luminosities only slightly greater than the catalogue limit. This means that some candidate isolated galaxies actually have nearby neighbours of quite similar luminosity. Reda et al. (2004) defined a 36 nearby isolated early-type galaxies, his isolation criteria have no comparable-mass neighbours within radial velocities $\mathrm{V}<9000 \mathrm{~km} \mathrm{~s}^{-1}$, 2 B-band magnitudes, $0.67 \mathrm{Mpc}$ in the plane of the sky and $700 \mathrm{~km} \mathrm{~s}^{-1}$ in recession velocity. Karachentseva et al. (2010) presented a list of 75 isolated latetype dwarf galaxies with criteria have no neighbors with a relative radial velocity difference of less than $500 \mathrm{~km} / \mathrm{s}$ or projected separations within $500 \mathrm{kpc}$. These were selected from $\sim 2000 \mathrm{dwarf}$ galaxies with radial velocities in Local Group $V_{L G}<3500 \mathrm{~km} / \mathrm{s}$ within the volume of the Local super cluster. In previous studies of the deviation of the light profiles, Burkert (1993) has studied the intermediate axis surface brightness distribution of elliptical galaxies, He has shown that the $\mathrm{r}^{1 / 4}$ law provides an excellent fit to the observed brightness distribution within the radius range $0.1 R_{e} \leq R \leq 1.5 R_{e}$, with mean deviations $\left\langle\delta \mu>\right.$ smaller than 0.1 mag $\operatorname{arcsec}^{-2}$ and the maximum deviations smaller than $0.2 \mathrm{mag} \operatorname{arcsec}^{-2}$. Younis $(2000 \mathrm{a}, \mathrm{b})$ has studied the systematic deviation from the $\mathrm{r}^{1 / 4}$ - law for two samples of elliptical galaxies and he 
found that the mean deviation are less than $0.12 \mathrm{mag} \operatorname{arcsec}^{-2}$, for one sample and less than 0.09 mag. $\operatorname{arcsec}^{-2}$ for the other. Recently, Younis and Basil (2011), have studied the surface brightness profiles of 46 elliptical galaxies, belong to Coma Cluster of galaxies have been fitted by $\mathrm{r}^{1 / \mathrm{n}}$ - law for the intermediate radius range $0.1 R_{e} \leq R \leq 1.5 R_{e}$. The mean deviations $<\delta \mu>$ found to be less than $0.03 \mathrm{mag} \operatorname{arcsec}^{-2}$. The deviation profiles between the observations and the adopted models show that the maximum negative deviation found to be around the reduced radius of 0.86 , while the maximum positive deviation were found to be around the reduced radius of 0.96 . The crossing points between the adopted models and the observed surface brightness profiles were found to be around the reduced radius $0.65,0.83$ and 1.0 .

In this work, has been adopted the Deviations of the Surface brightness distribution in Isolated Elliptical galaxies to drive the photometry parameter, were analyzed the surface brightness distribution of 48 isolated elliptical galaxies with the model proposed by $\mathrm{r}^{1 / n}-$ law for the radial range $0.1 \leq\left(\frac{R}{R_{e}}\right) \leq 1.5,43$ galaxy selected from data published by Fasano and Bonoli, (1989) and 5 galaxy from Reda et al. (2004).

\section{Data selection and Reduction:}

Our sample content a 43 galaxy was selected from a published paper by (Fasano \& Bonoli, 1989), these galaxies was selected from the UGC (Uppsala General Catalog of Galaxies) catalogue (Nilson, 1973), the galaxies are calssified as ellipticals in catalogue. The observation were done with $1.82 \mathrm{~m}$ telescope in Asiago (Ekar), Italy. The scale (CDD) was 0.290 arcsec.pixel $^{-1}$ and the filed size format of $385 \times 578$.

For the 5 galaxy from Reda et al. (2004), candidate isolated earlytype galaxies was taken from the Lyon-Meudon Extragalactic Data Archive (LEDA). From this catalogue Reda selected galaxies that the following criteria: (i) morphological type $\mathrm{T} \leq-3$; (ii) Virgo corrected recession velocity $\mathrm{V} \leq 9000 \mathrm{~km} \mathrm{~s}^{-1}$; (iii) apparent magnitude $\mathrm{B} \leq 14.0$; the observation of galaxies in the B- band and R-band images were obtained using the $3.9 \mathrm{~m}$ Anglo-Australian Telescope, with a pixel scale of 0.229 arcsec.pixel ${ }^{-1}$ giving a field of view of $30.6 \times 30.6 \mathrm{arcmin}^{2}$.

Figure (2) shows the distribution of these galaxies as a function of their absolute magnitude $\mathrm{M}_{\mathrm{B}}$. Here in this papers, it has adopted the Sersic law $\left(r^{1 / n}-\right.$ law) to determine the fitting between $r^{1 / n}-$ law and the observed surface brightness profiles of the adopted sample of isolated elliptical galaxies for the intermediate radial range $0.1 \leq\left(\frac{R}{R_{e}}\right) \leq 1.5$, this range cover more than $60 \%$ of the total luminous mass which provides a 
good representation of the distribution of the visible matter (Burket 1993).

The effective radius $R_{\mathrm{e}}$ for the adopted radial range was determined in a self-consistent method. The first estimated value for the $\mathrm{R}_{\mathrm{e}}$ was derived from the $\mathrm{r}^{1 / \mathrm{n}}$ - law using the lsqcurvefit function of the MatLab package.

$\mu(x)=\mu_{o}+1.0857 b_{n}\left(\frac{R}{R_{e}}\right)^{1 / n}$

Where $\quad b_{n} \cong 2 n-0.327, \quad \mu_{o}=\mu_{e}-1.0857 b_{n}$

For this new value of $\mathrm{R}_{\mathrm{e}}$ a new range of radius is updated as $0.1 R_{e}^{1 / n} \leq R^{1 / n} \leq 1.5 R_{e}^{1 / n}$ then the procedure of the fitting is repeated to updated the value of $\mathrm{R}_{\mathrm{e}}$ and then the range of radius until converged $\mathrm{R}_{\mathrm{e}}$ was obtained. For each galaxy of the adopted sample, the deviation profiles $\delta \mu(\mathrm{x})$ from the best fitting $\mathrm{r}^{1 / \mathrm{n}}$ - law and the mean deviation $\langle\delta \mu>$ were determined using the following two equations

$$
\begin{aligned}
& \delta \mu(x)=\mu(x)-\mu_{s}(x) \\
& <\delta \mu>=\frac{1}{\sqrt{N}}\left[\sum_{i}^{N}\left(\mu\left(x_{i}\right)-\mu_{s}\left(x_{i}\right)\right)^{2}\right]^{\frac{1}{2}}
\end{aligned}
$$

Where $\mu(\mathrm{x})$ is the observed surface brightness profiles, $\mu_{\mathrm{s}}(\mathrm{x})$ is the best fitting $\mathrm{r}^{1 / n}$ - law to the surface brightness profiles, and $\mathrm{N}$ is the total number of the data points within the radial range

\section{Results and Discussion:}

Figure (1) shows the observed surface brightness profiles, the best fitting $\mathrm{r}^{1 / n}$ - law, and their deviation from its models for the 48 isolated elliptical galaxies. All galaxies of the sample in figure (1) contains a top and bottom figure. The top of the figure shows the surface brightness and their best fitting and bottom figure shows the deviations from the $r^{1 / n}-$ law, the deviation profiles shows $65 \%$ of the sample have a negative deviation (i.e. the surface brightness of the galaxy brighter than $r^{1 / n}-$ law) and $35 \%$ of the sample have a positive deviation (i.e. the surface brightness of the galaxy fainter than $\mathrm{r}^{1 / \mathrm{n}}$ - law) at the inner parts of galaxy (small radii). The derived parameters are listed within table (1): Column 1 - 13, name of galaxy, type of galaxy, absolute magnitude $\left(\mathrm{M}_{\mathrm{B}}\right)$, mean deviation $\left\langle\delta \mu>\right.$, effective radius of the intermediate range radii $\left(\mathrm{R}_{\mathrm{e}}\right)$, shape parameter $(\mathrm{n})$, the radial position at reduce radius of the first crossing point $\left(\mathrm{r}_{\mathrm{C} 1}^{\prime}\right)$, the radial position of the second crossing point $\left(\mathrm{r}_{\mathrm{C} 2}^{\prime}\right)$, the radial position of the third crossing point $\left(\mathrm{r}_{\mathrm{C} 3}^{\prime}\right)$, the position of the top of the hump $\left(\mathrm{r}_{\mathrm{p}+}^{\prime}\right)$, the maximum positive deviation $\left(\mathrm{p}_{+}\right)$, the position of the bottom of the dip $\left(\mathrm{r}_{\mathrm{p}-}^{\prime}\right)$, and the last column is maximum negative deviation ( $\mathrm{p}_{-}$). 
Figure (3) shows the distribution of the isolated elliptical galaxies as a function of their mean deviation $\langle\delta \mu>$, the most of the galaxies were found to be less than (0.035) mag. $\operatorname{arcsec}^{-2}$. Figure (4) shows the position of the top of the hump of the deviation profiles for the sample which is found at the reduced radius $r_{p^{+}}^{\prime}=0.95$, while figure (5) shows the bottom of the dip of the deviation profiles for the sample of galaxies which is found to be at the reduced radius $r_{p-}^{\prime}=0.81$. The radial position of the crossing points $\left(r_{c}^{\prime}\right)$ (i.e. the points at which the deviations change their signs) have been found for each galaxy. The first crossing points, the second and the third, for all galaxies of the sample found to be around the reduced radius $r_{\mathrm{C}_{1}}^{\prime} \cong 0.63, \mathrm{r}_{\mathrm{C} 2}^{\prime} \cong 0.8$ and $\mathrm{r}_{\mathrm{C}_{3}}^{\prime} \cong 0.95$ see figure $(6,7$, and 8$)$.

Although the sample that chosen in this study, consists of isolated galaxies, the results showed that the deviations between the observed and the models proposed for the sample's galaxies found to be similar to the previous results, therefore the deviations do not depend on the surrounding environment of the galaxies.

\section{References:}

1) Burkert, A., (1993) "Do Elliptical Galaxies Have $r^{1 / 4}$ Brightness Profiles?". Astro. \& Astrophys., 278, 23-28.

2) Caon, N., Capaccioli, M., D'Onofrio, M., (1993) "On The Shape Of The Light Profiles Of Early-Type Galaxies". Mon. Not. Roy. Astro. Soc., 256, 1013.

3) Colbert, J. W.; Mulchaey, J. S.; Zabludoff, A. I. (2001), "The Optical and Near-Infrared Morphologies of Isolated Early-Type Galaxies", A. J., 121, 808C.

4) De Vaucouleurs, G., De Vaucouleurs, A., Corwin JR., Buta R. Paturel G. and Fouque P. (1991) "third Reference Catalogue of Bright galaxies". RC3, M 0000d.

5) Fasano, G. \& Bonoli, C. (1989) "Isophotal twisting in isolated elliptical galaxies " A\&AS, 79, 291F.

6) Jack Sulentic, (2009), "Isolated Galaxies History of Research and Ideas Over the Past 40 Years", International Conference on Isolated Galaxies, May $12^{\text {th }}-15^{\text {th }}$, Granada, Spain.

7) Karachentseva V. E., (1973), "The Catalogue of Isolated Galaxies" Astrof. Issledovanija Byu. Spec. Ast. Obs., 8, 3 k.

8) Karachentseva V. Karachentsev I., Sharina M., (2010), "isolated dwarf galaxies in the local supercluster and its surroundings", Astrophysics, Vol. 53, No. 4.

9) Nilson, P., (1973), "Uppsala General Catalogue Of Galaxies" Acta Universitatis Upsalienis, Nova Regiae Societatis Uppsalienis, Series V: A Vol. 1. 
Deviations of the Surface Brightness Distribution in Isolated Elliptical Galaxies.

10) Pamela M., Christian E., Michael N., 2004, "early-type galaxies in extremely isolated environments: typical ellipticals?", Astro. J. 127: p3213-3234.

11) Reda, F., Forbes. D., Beasley, M., O'Sullivan, E., Goudfrooij, P. (2004), "The photometric properties of isolated early-type galaxies", Mon. Not. Roy. Astro. Soc., 354, 851R

12) Sersic, J. L., (1968) Atlas de Galaxias Australes (Cordoba: Observatorio Astronomico, Universidad de Cordoba)

13) Trujillo, I., Aguerri, J. A. L., Cepa, J., Gutierrez, C. M., (2001) "The Effects of Seeing On Sersic Profiles". Mon. Not. Roy. Astro. Soc., 321, 269-276.

14) Younis, S. M., (2000a) "Systematic Deviations From $r^{1 / 4}$ ed Vaucouleurs Law In Elliptical Galaxies". J. Edu. Sci., 41.

15) Younis, S. M., (2000b) "Surface Brightness Profiles In Early-Type Galaxies: A Study Of The Small Deviations From The $r^{1 / 4}$ Law". J. Edu. Sci., 42, 77.

16) Younis, S. M., Basil O. M., (2011) "Deviations of the Light Distribution from the Sersic's model in Elliptical galaxies". J. Edu. Sci., 24.

17) Zwicky F. et al. (1957), "Catalog of Galaxies and Clusters of Galaxies" V.1 1-6 California Inst. Tech. Pasadena.

Table (1)

\begin{tabular}{|c|c|c|c|c|c|c|c|c|c|c|c|c|}
\hline galaxy & Type* $^{*}$ & $\begin{array}{c}-\mathbf{M}_{\mathbf{B}}{ }^{*} \\
\text { (mag.) }\end{array}$ & $\begin{array}{c}<\delta \mu> \\
\text { mag } \\
\operatorname{arcsec}^{-2} \\
\end{array}$ & $\begin{array}{c}\mathbf{X}_{\mathbf{e}} \\
\operatorname{arcsec}\end{array}$ & $\mathbf{n}$ & $\mathbf{r}_{\mathrm{C} 1}^{\prime}$ & $\mathbf{r}_{\mathrm{C} 2}^{\prime}$ & $\mathbf{r}_{\mathrm{C} 3}$ & $\mathbf{r}_{\mathbf{P}+}$ & $\mathbf{P +}$ & $r_{P-}^{\prime}$ & P- \\
\hline NGC 7796 & E (cD) & 20.93 & 0.0270 & 45.049 & 5.662 & 0.667 & 0.740 & 0.844 & 0.699 & 0.0416 & 0.789 & -0.0289 \\
\hline ESO 318-G021 & E? & 20.94 & 0.0279 & 35.109 & 7.956 & 0.694 & 0.777 & 0.863 & 0.817 & 0.0384 & 0.733 & -0.0387 \\
\hline NGC 2865 & E3-4 & 20.83 & 0.0231 & 21.069 & 3.891 & 0.671 & 0.843 & 1.027 & 0.773 & $\mathbf{0 . 0 3 3 0}$ & 0.917 & -0.0349 \\
\hline NGC 821 & E6 & 20.82 & 0.0356 & 5.223 & 1.880 & 0.485 & 0.779 & 1.134 & 0.955 & $\begin{array}{c}0.0478 \\
\end{array}$ & 1.231 & $\begin{array}{c}-0.0739 \\
\end{array}$ \\
\hline ESO 153-G003 & $\mathbf{E}$ & 21 & 0.0183 & 54.028 & 8.398 & 0.739 & 0.779 & 0.837 & 0.940 & $\mathbf{0 . 0 3 0 0}$ & 0.869 & -0.0284 \\
\hline UGC 236 & $\mathbf{E}$ & 19.88 & 0.0204 & 5.336 & 2.693 & 0.682 & 0.813 & 0.964 & 0.890 & $\mathbf{0 . 0 3 4 0}$ & 0.766 & -0.0361 \\
\hline UGC 741 & $\mathbf{E}$ & 20.84 & 0.0281 & 8.279 & 5.121 & 0.732 & 0.818 & 0.921 & 0.868 & 0.0430 & 0.785 & -0.0379 \\
\hline UGC 881 & $\mathbf{E}$ & 20.97 & 0.0140 & 13.805 & 4.863 & 0.776 & 0.871 & 0.959 & 0.909 & 0.0206 & 1.036 & -0.0226 \\
\hline UGC 1030 & $\mathbf{E}$ & 19.23 & 0.0293 & 6.783 & 2.288 & 0.589 & 0.899 & 1.125 & 0.982 & 0.0494 & 1.215 & -0.0464 \\
\hline UGC 1043 & $\bar{E}$ & 19.26 & 0.0375 & 8.599 & 2.279 & 0.673 & 0.887 & 1.098 & 0.974 & 0.0702 & 0.788 & -0.0503 \\
\hline UGC 1503 & $\mathbf{E}$ & 20.46 & 0.0302 & 8.218 & 1.147 & 0.541 & 0.847 & 1.141 & 1.289 & 0.0463 & 0.749 & -0.0416 \\
\hline UGC 1543 & $\overline{\mathbf{E}}$ & 21.07 & 0.0378 & 19.304 & 3.522 & 0.561 & 0.779 & 0.947 & 1.079 & 0.0446 & 0.859 & -0.0602 \\
\hline UGC 1788 & $\mathbf{E}$ & 22.05 & 0.0258 & 13.706 & 2.496 & 0.577 & 0.677 & 0.799 & 1.067 & 0.0406 & 0.985 & -0.0484 \\
\hline UGC 2022 & $\mathbf{E}$ & 19.54 & 0.0403 & 7.968 & 2.515 & 0.502 & 0.679 & 0.855 & 0.797 & 0.0415 & 0.577 & -0.0870 \\
\hline UGC 2656 & $\mathbf{E}$ & 19.87 & $\begin{array}{l}0.0398 \\
\end{array}$ & 9.513 & 2.808 & 0.536 & 0.674 & 0.836 & 0.603 & 0.0643 & 0.754 & -0.0508 \\
\hline UGC 2717 & $\mathbf{E}$ & 20.13 & 0.0126 & 17.523 & 6.215 & 0.741 & 0.822 & 0.901 & 0.975 & 0.0291 & 0.960 & -0.0218 \\
\hline UGC 2957 & $\mathbf{E}$ & 21.12 & 0.0151 & 231.43 & $\begin{array}{c}11.21 \\
5 \\
\end{array}$ & 0.630 & 0.673 & 0.726 & 0.702 & 0.0317 & 0.663 & -0.0205 \\
\hline UGC 3307 & $\mathbf{E}$ & 19.23 & 0.0375 & 9.615 & 4.574 & 0.657 & 0.895 & 1.024 & 0.961 & 0.0601 & 1.052 & -0.0619 \\
\hline UGC 3646 & $\mathbf{E}$ & 21.41 & 0.0399 & 15.605 & 4.080 & 0.593 & 0.759 & 0.943 & 0.651 & 0.0586 & 0.864 & -0.0491 \\
\hline UGC 4782 & $\mathbf{E}$ & 21.31 & 0.0319 & 6.206 & 1.626 & 0.676 & 0.859 & 1.202 & 1.374 & 0.0438 & 0.753 & -0.0359 \\
\hline UGC 5705 & $\mathbf{E}$ & 17.90 & 0.0384 & 9.489 & 2.289 & 0.552 & 0.795 & 1.049 & 0.663 & 0.0591 & 0.908 & -0.0585 \\
\hline
\end{tabular}


Omar Basil Mohammad Saleh

\begin{tabular}{|c|c|c|c|c|c|c|c|c|c|c|c|c|}
\hline galaxy & Type $^{*}$ & $\begin{array}{c}-\mathbf{M}_{\mathbf{B}}{ }^{*} \\
\text { (mag.) }\end{array}$ & $\begin{array}{c}<\delta \mu> \\
\text { mag } \\
\operatorname{arcsec}^{-2} \\
\end{array}$ & $\begin{array}{c}\mathbf{X}_{\mathbf{e}} \\
\operatorname{arcsec}\end{array}$ & $\mathbf{n}$ & $\mathbf{r}_{\mathrm{C} 1}^{\prime}$ & $\mathbf{r}_{\mathrm{C} 2}^{\prime}$ & $r_{\mathrm{C} 3}^{\prime}$ & $\mathbf{r}_{\mathbf{P}+}^{\prime}$ & $\mathbf{P +}$ & $\mathbf{r}_{\mathrm{P}-}^{\prime}$ & P. \\
\hline UGC 5955 & $\mathbf{E}$ & 17.30 & 0.0138 & 9.476 & 2.776 & 0.602 & 0.782 & 0.945 & 1.076 & 0.0170 & 1.124 & -0.0168 \\
\hline UGC 6159 & $\mathbf{E}$ & 17.49 & 0.0229 & 206.13 & $\begin{array}{c}12.52 \\
9 \\
\end{array}$ & 0.688 & 0.713 & 0.754 & 0.732 & 0.0396 & 0.699 & -0.0350 \\
\hline UGC 6504 & $\mathbf{E}$ & 21 & 0.0375 & 10.824 & 4.381 & 0.649 & 0.805 & 1.011 & 0.936 & 0.0415 & 0.702 & -0.0626 \\
\hline UGC 6671 & $\mathbf{E}$ & 21.48 & 0.0358 & 4.994 & 2.518 & 0.588 & 0.753 & 0.966 & 0.848 & 0.0376 & 0.602 & -0.0588 \\
\hline UGC 6810 & $\mathbf{E}$ & 20.64 & 0.0211 & 12.853 & 6.191 & 0.710 & 0.923 & 1.012 & 1.032 & 0.0190 & 0.977 & -0.0248 \\
\hline UGC 7115 & $\mathbf{E}$ & 20.87 & 0.0425 & 8.731 & 5.189 & 0.733 & 0.872 & 1.006 & 0.933 & 0.0460 & 0.759 & -0.0533 \\
\hline UGC 7224 & $\mathbf{E}$ & 20.72 & 0.0276 & 22.898 & 7.049 & 0.699 & 0.763 & 0.631 & 0.924 & 0.0374 & 0.965 & -0.0480 \\
\hline UGC 7681 & $\mathbf{E}$ & 18.73 & 0.0197 & 17.153 & 1.835 & 0.454 & 0.743 & 1.021 & 0.906 & 0.0301 & 0.574 & -0.0249 \\
\hline UGC 7767 & $\bar{E}$ & 18.11 & 0.0350 & 5.444 & 2.461 & 0.579 & 0.783 & 1.039 & 0.882 & $\begin{array}{c}0.0343 \\
\end{array}$ & 1.095 & -0.0479 \\
\hline UGC 7838 & $\mathbf{E}$ & 19.72 & 0.0334 & 14.714 & 1.447 & 0.380 & 0.655 & 1.014 & 0.771 & 0.0473 & 0.753 & -0.0484 \\
\hline UGC 7988 & $\mathbf{E}$ & 21.21 & 0.0236 & 12.101 & 3.897 & 0.616 & 0.704 & 0.850 & 0.929 & 0.0324 & 0.785 & -0.0291 \\
\hline UGC 8376 & $\mathbf{E}$ & 23.09 & 0.0296 & 9.955 & 2.888 & 0.545 & 0.736 & 0.976 & 0.849 & 0.0311 & 0.611 & -0.0475 \\
\hline UGC 8453 & $\mathbf{E}$ & 22.64 & $\mathbf{0 . 0 3 2 7}$ & 13.169 & 5.138 & 0.661 & 0.749 & 0.893 & 0.815 & 0.0462 & 0.962 & -0.0485 \\
\hline UGC 8779 & $\mathbf{E}$ & 22.74 & 0.0250 & 11.279 & 4.126 & 0.566 & 0.664 & 0.814 & 0.731 & 0.0364 & 0.592 & -0.0404 \\
\hline UGC 9070 & $\mathbf{E}$ & 18.13 & 0.0228 & 25.573 & 4.564 & 0.629 & 0.784 & 0.936 & 0.957 & 0.0588 & 0.887 & -0.0326 \\
\hline UGC 9278 & $\mathbf{E}$ & 20.32 & 0.0380 & 11.752 & 4.970 & 0.638 & 0.715 & 0.819 & 0.981 & 0.0597 & 0.874 & -0.0518 \\
\hline UGC 10103 & $\mathbf{E}$ & 20.63 & 0.0347 & 6.043 & 4.188 & 0.691 & 0.849 & 0.992 & 1.144 & 0.0391 & 0.728 & -0.0613 \\
\hline UGC 10352 & $\mathbf{E}$ & 20.98 & 0.0258 & 2.798 & 1.784 & 0.643 & 0.785 & 0.911 & 0.806 & 0.0313 & 0.757 & -0.0532 \\
\hline UGC 10410 & $\mathbf{E}$ & 21.25 & 0.0347 & 12.288 & 4.329 & 0.625 & 0.731 & 0.911 & 0.793 & 0.0329 & 0.665 & -0.0658 \\
\hline UGC 10467 & $\mathbf{E}$ & 19.70 & 0.0169 & 12.570 & 2.873 & 0.621 & 0.869 & 1.109 & 1.184 & 0.0227 & 1.019 & -0.0250 \\
\hline UGC 10595 & $\bar{E}$ & 18.71 & 0.0230 & 14.968 & 5.603 & 0.709 & 0.811 & 0.929 & 0.755 & 0.0412 & 0.866 & $\begin{array}{c}-0.0278 \\
\end{array}$ \\
\hline UGC 10695 & $\mathbf{E}$ & 22.84 & 0.0179 & 25.015 & 3.736 & 0.559 & 0.732 & 0.959 & 1.011 & 0.0253 & 0.886 & -0.0175 \\
\hline UGC 10827 & $\mathbf{E}$ & 21.22 & 0.0283 & 23.688 & 6.279 & 0.614 & 0.686 & 0.843 & 0.814 & 0.0201 & 0.629 & -0.0392 \\
\hline UGC 10836 & $\mathbf{E}$ & 21.39 & 0.0188 & 32.874 & 5.655 & 0.613 & 0.676 & 0.761 & 0.717 & 0.0292 & 0.639 & -0.0313 \\
\hline UGC 11605 & $\overline{\mathbf{E}}$ & 20.83 & 0.0358 & 20.868 & 5.601 & 0.652 & 0.765 & 0.920 & 0.869 & 0.0246 & 0.685 & -0.0503 \\
\hline UGC 12002 & $\mathbf{E}$ & 19.86 & 0.0231 & 13.177 & 3.032 & 0.557 & 0.803 & 1.079 & 1.100 & 0.0417 & 0.910 & $-\mathbf{0 . 0 3 0 7}$ \\
\hline UGC 12733 & $\mathbf{E}$ & 21.57 & 0.0231 & 25.989 & 4.114 & 0.574 & 0.721 & 0.806 & 0.679 & 0.0291 & 0.773 & -0.0428 \\
\hline
\end{tabular}

\section{4}


Deviations of the Surface Brightness Distribution in Isolated Elliptical Galaxies.
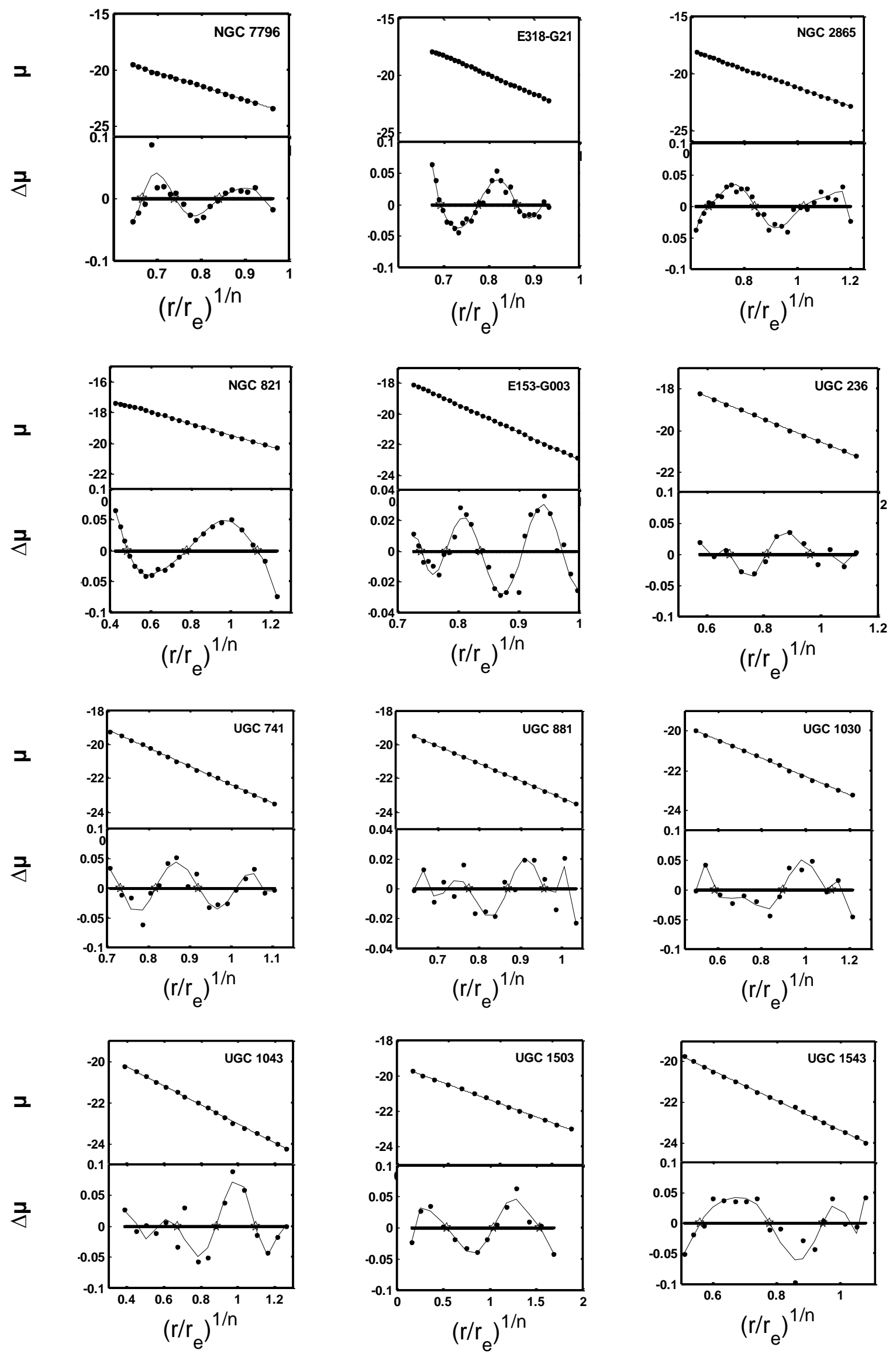

Figure (1): represent the surface brightness profiles $(\mu)$ and its deviations $(\Delta \mu)$ agents radius range of $\mathbf{r}^{1 / n}$ for isolated elliptical galaxies: the point is the observations, solid line is the $\mathbf{r}^{1 / n}$ - law 


\section{Omar Basil Mohammad Saleh}
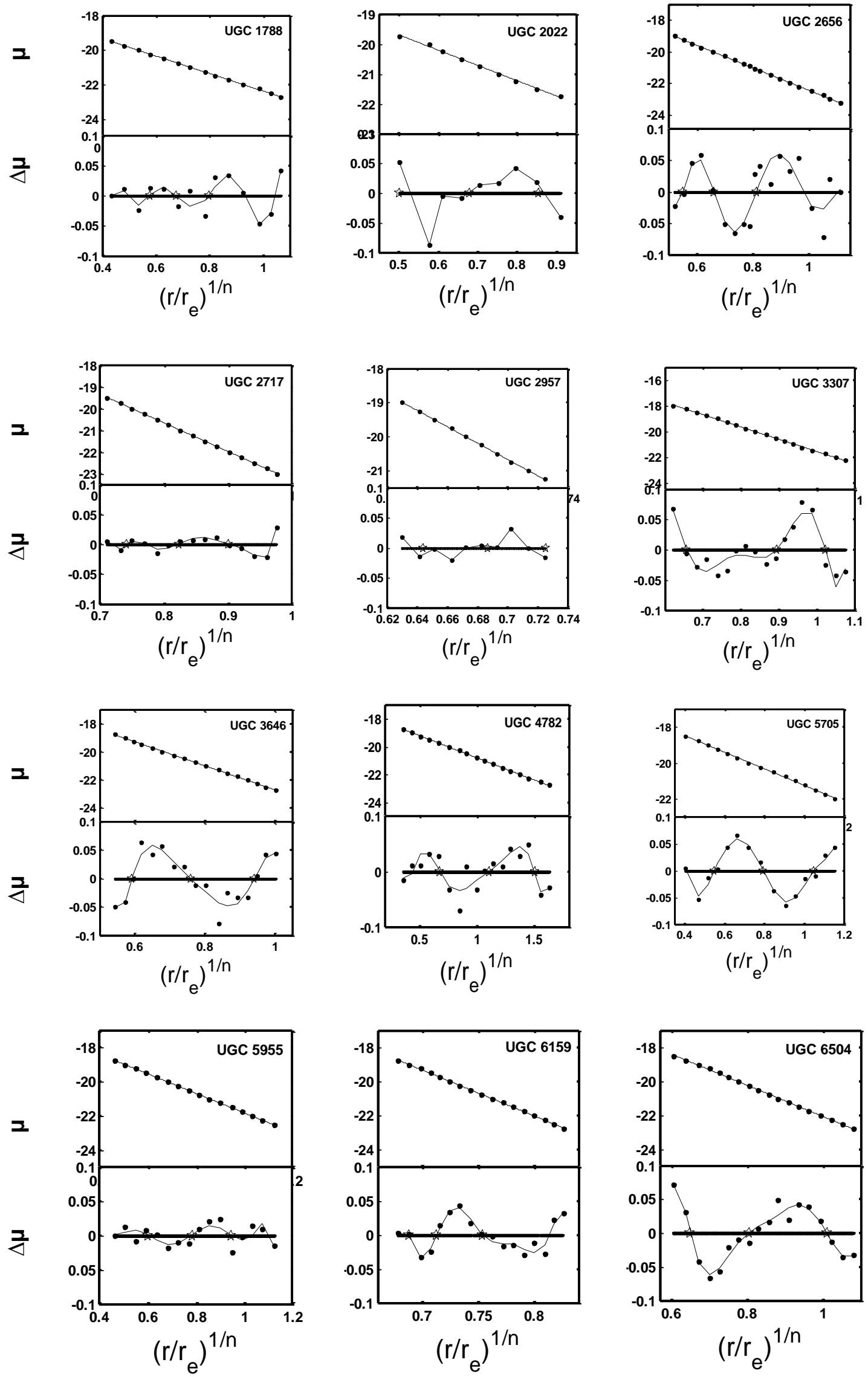

Complement - Figure (1) 
Deviations of the Surface Brightness Distribution in Isolated Elliptical Galaxies.
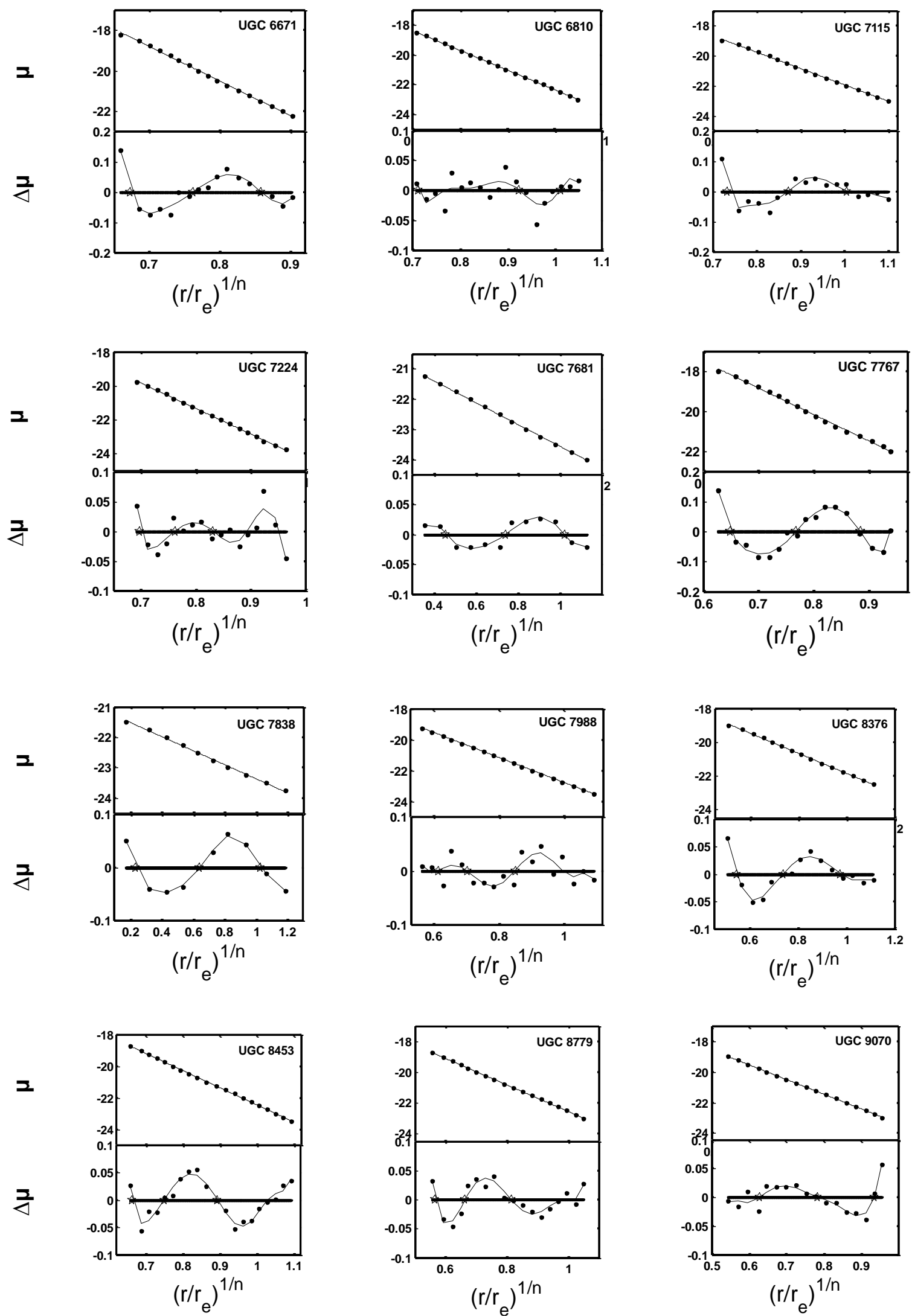

Complement - Figure (1) 

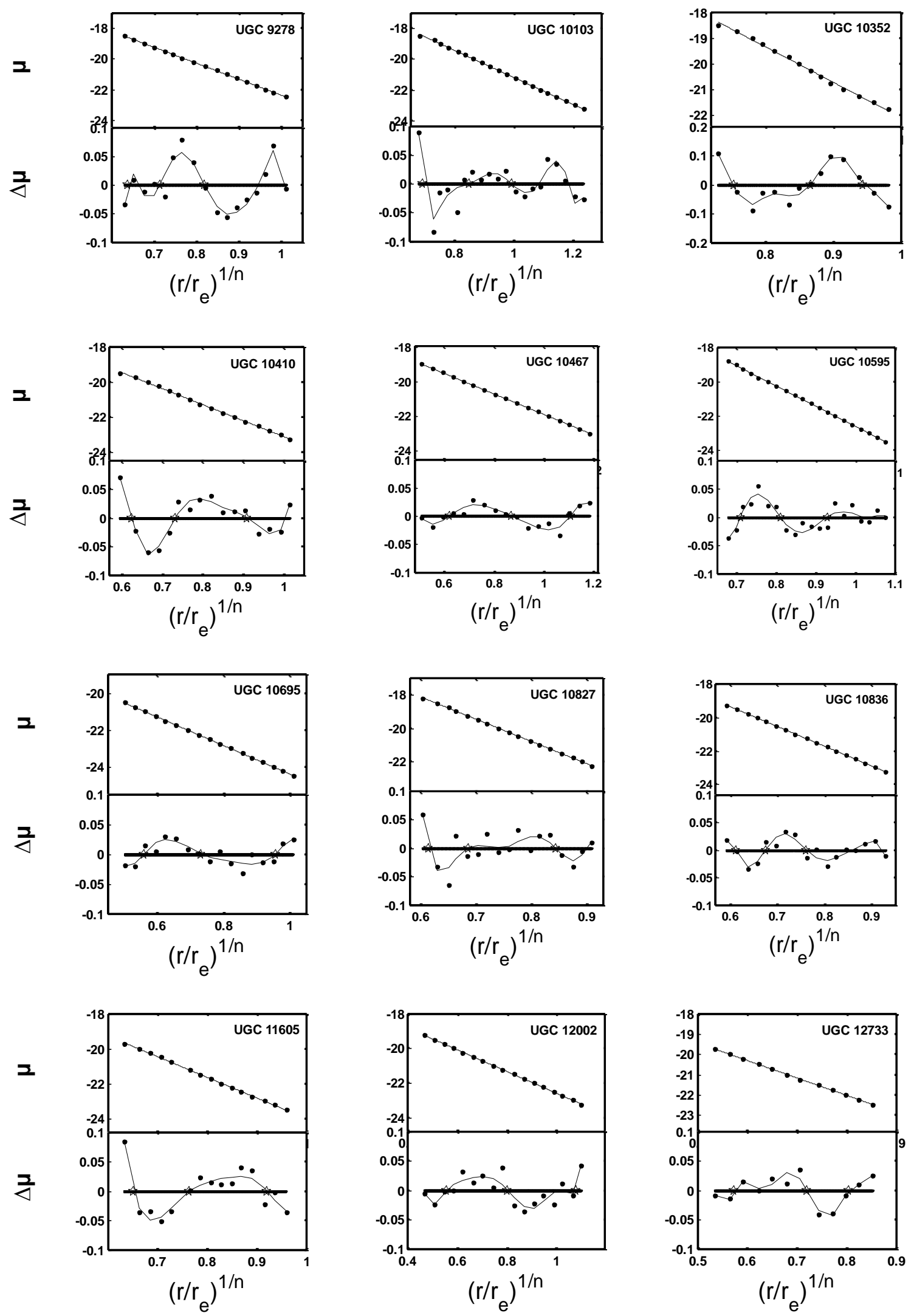

Complement - Figure (1) 
Deviations of the Surface Brightness Distribution in Isolated Elliptical Galaxies.

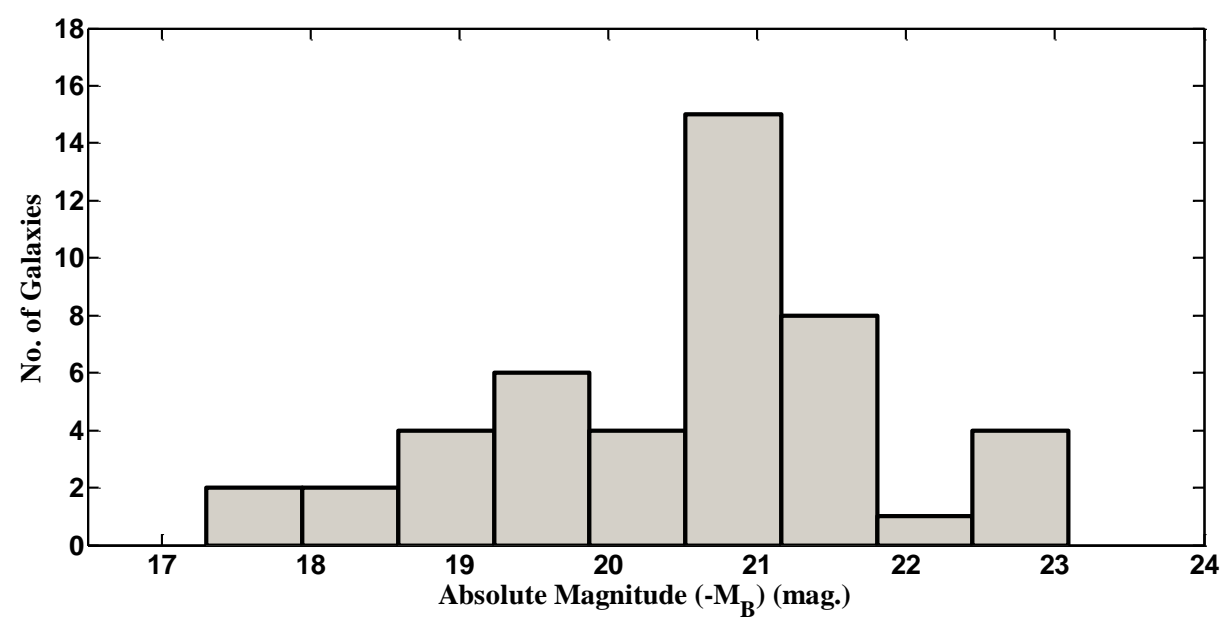

Figure (2): The distribution of the isolated elliptical galaxies as a function of their absolute magnitude

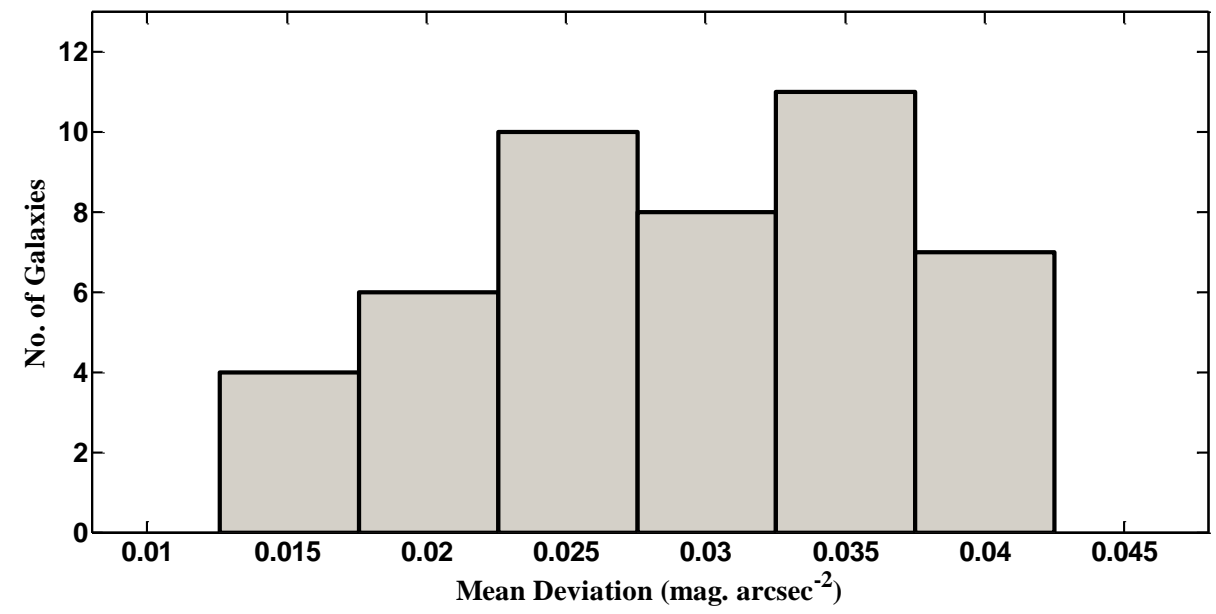

Figure (3): the number of isolated Elliptical galaxies is shown as a function of their mean deviations $<\delta \mu>$

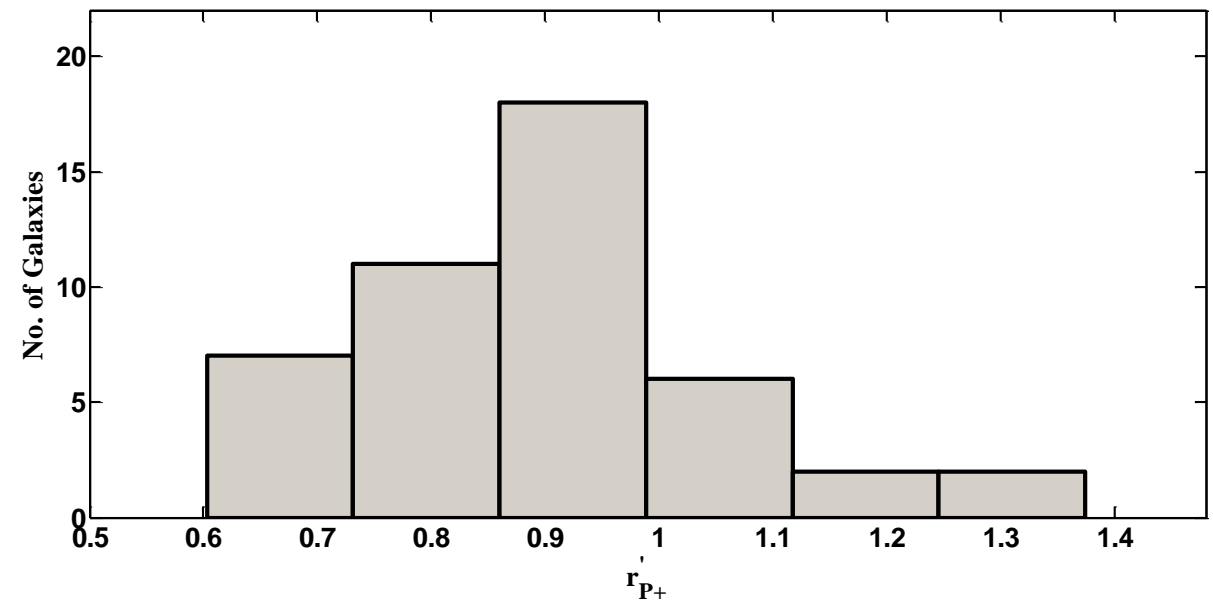

Figure (4): The radial position of the maximum positive deviation (top of the hump) $\mathbf{r}^{\prime} \mathbf{P}+$ 


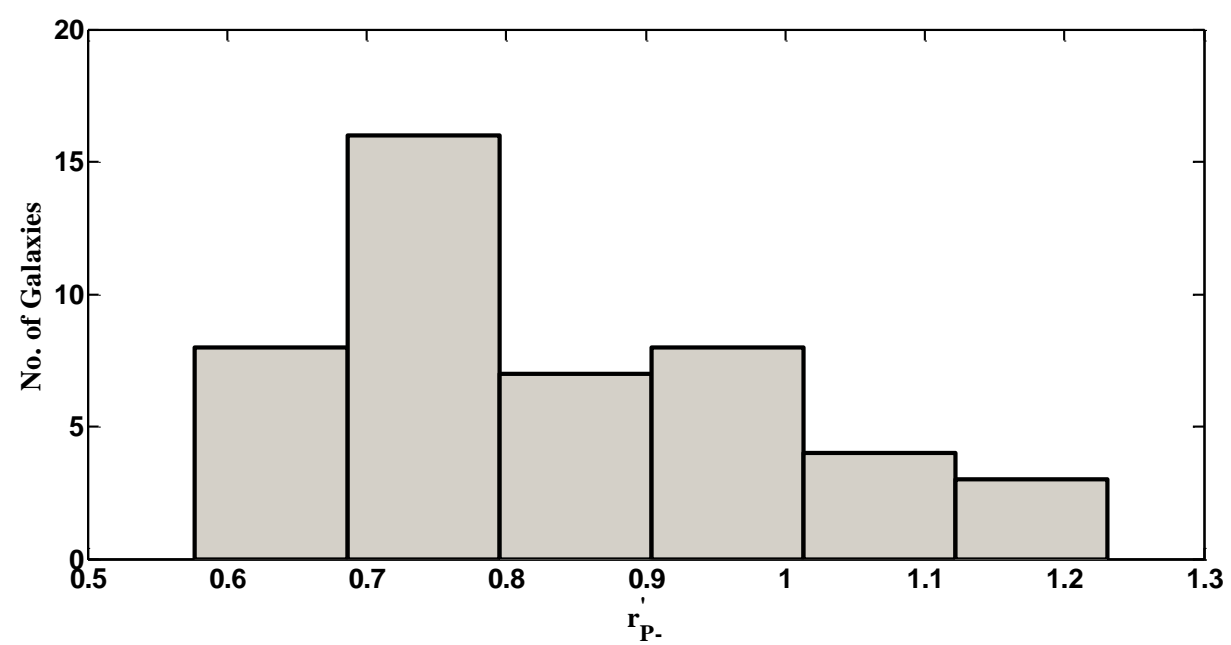

Figure(5): The radial position of the maximum negative deviation (Lower point) $\mathbf{r}^{\prime} \mathbf{P}$ -

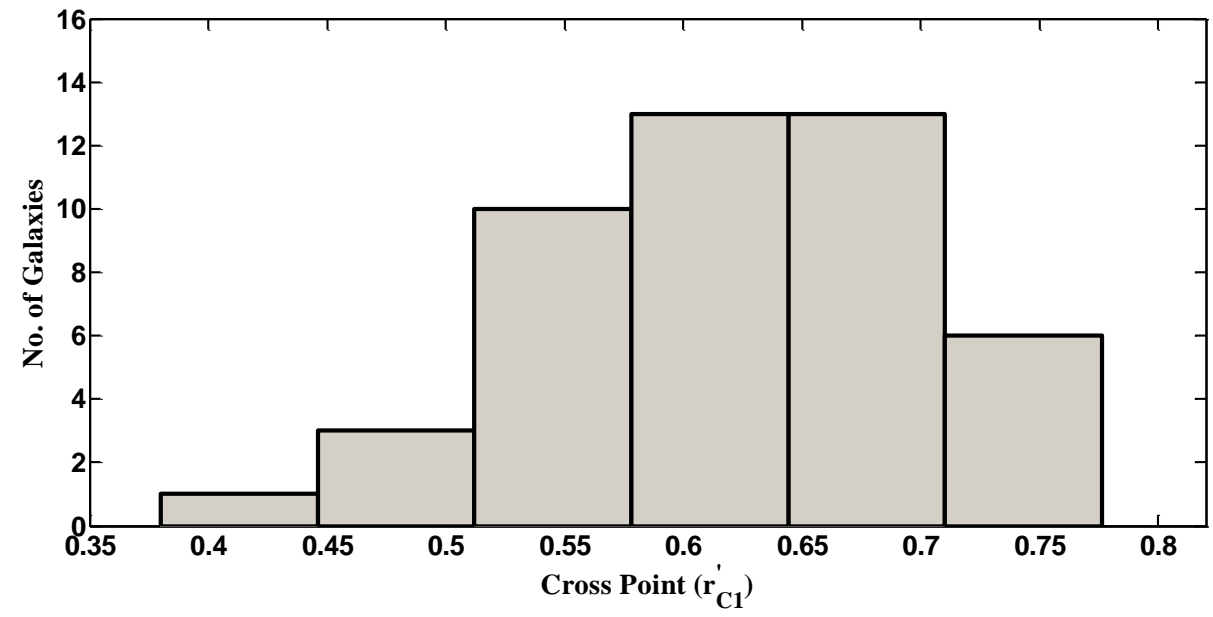

Figure (6): The radial position of $\mathbf{r}^{\prime} \mathrm{C} 1$

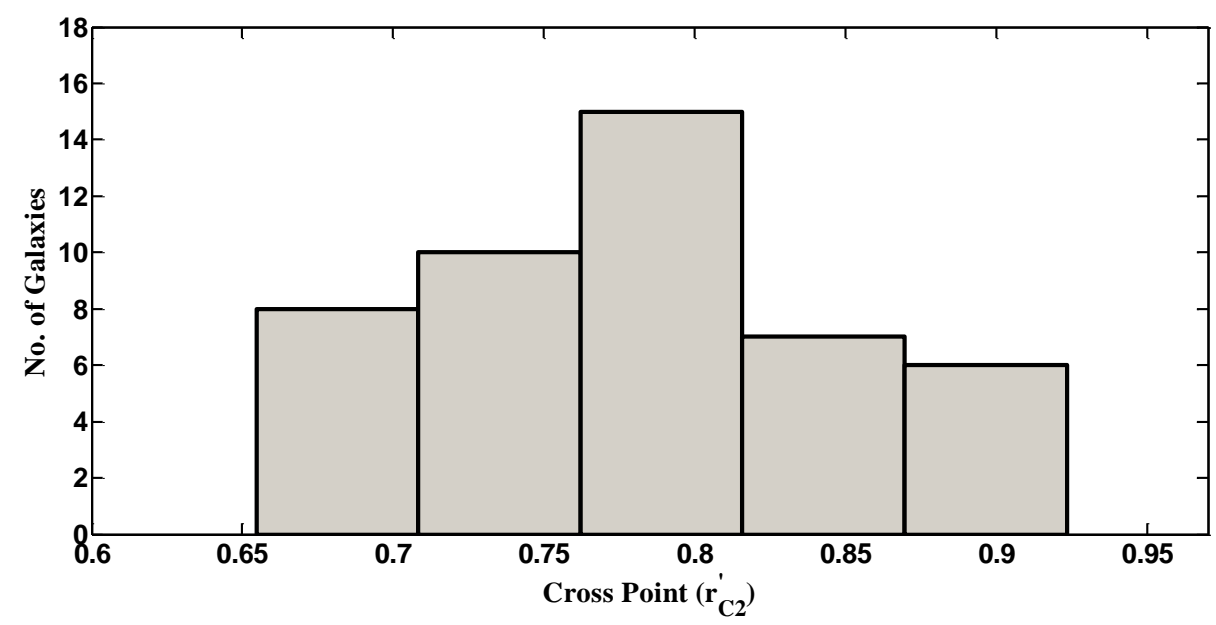

Figure (7): The radial position of $\mathbf{r}^{\prime} \mathrm{c} 2$ 


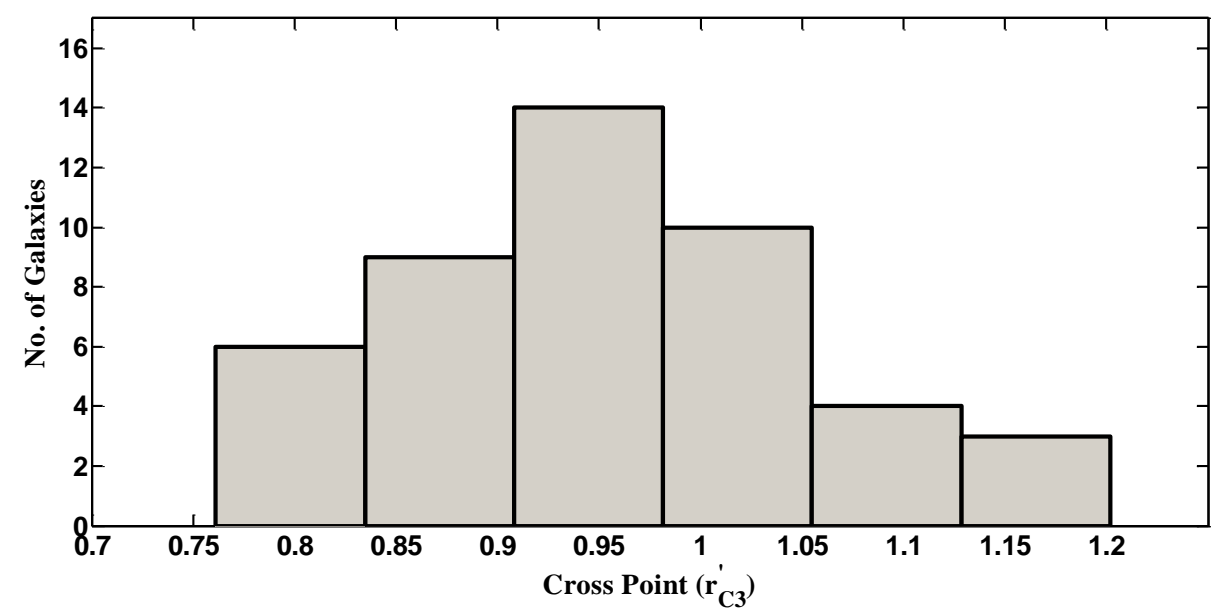

Figure(8): The radial position of $\mathbf{r}^{\prime} \mathrm{c3}$

Table (2)

\begin{tabular}{|c|c|c|c|c|c|}
\hline $\begin{array}{l}\text { Deviation } \\
\text { Parameters }\end{array}$ & $\begin{array}{c}\text { Burkert } \\
{(11993)^{*}}^{*}\end{array}$ & $\begin{array}{l}\text { Younis } \\
(\text { 2000a) }\end{array}$ & $\begin{array}{l}\text { Younis } \\
(2000 b)^{*}\end{array}$ & $\begin{array}{c}\text { Younis \& } \\
\text { Basil } \\
(2011)^{* *} \\
\end{array}$ & $\begin{array}{c}\text { The } \\
\text { present } \\
\text { research }\end{array}$ \\
\hline$<\delta \mu>$ & 0.1 & 0.12 & 0.09 & 0.03 & 0.035 \\
\hline $\mathrm{r}_{\mathrm{p}+}^{\prime}$ & --- & --- & 0.85 & 0.96 & 0.95 \\
\hline $\mathrm{r}_{\mathrm{p}-}^{\prime}$ & 0.8 & 0.8 & 0.75 & 0.83 & 0.81 \\
\hline$r_{\mathrm{C} 1}^{\prime}$ & --.- & --.- & --.- & 0.65 & 0.63 \\
\hline$r_{C 2}^{\prime}$ & ---- & ---. & ---- & 0.83 & 0.8 \\
\hline$r_{C 3}^{\prime}$ & -..- & ---- & -.-. & 1.0 & 0.95 \\
\hline
\end{tabular}

* = Deviation Parameters were derived by $\mathbf{r}^{1 / 4}$ - law

** = Deviation Parameters were derived by $\mathbf{r}^{1 / \mathbf{n}}$ - law 